Pak. J. Sci. Ind. Res. Ser. A: Phys. Sci. 201154 (1) 48-51

\title{
Contribution of Different Global Varieties of Cotton towards Water Hardness in Textile Wet Processing
}

\author{
Mumtaz Hasan Malik*, Tanveer Hussain and Aroosa Shaukat \\ National Textile University, Faisalabad-37610, Pakistan
}

(received November 16, 2009; revised July 23, 2010; accepted July 29, 2010)

\begin{abstract}
Specimens of nineteen different global varieties of cotton were studied to determine their contribution to water hardness through calcium and magnesium impurities, resulting in various problems during textile pretreatment, colouration and finishing. Pakistani cotton was found to be the second most contaminated cotton in terms of calcium and magnesium impurities, whereas Elisa variety from Uzbekistan was the cleanest.
\end{abstract}

Keywords. cotton, water hardness, magnesium, calcium

\section{Introduction}

Cotton is the backbone of the world's textile trade. It has many countless end uses, which make it one of the most abundantly used textile fibres in the world (Wilson, 2006; Yafa, 2006; Becerra, 2000; Cook, 1984). It is seed hair of plant of genus Gossypium (Lewin, 2006) and the purest form of cellulose found in nature. Although cotton may be as much as $96 \%$ cellulose, there are always some other components present in it as impurities. The level of impurities in cotton is affected by geology of the cultivation area, soil constitution, weather conditions during the maturing period, cultivation techniques, chemicals, pesticide, fertilisers and harvesting techniques. Among the impurities present in cotton, the elements that pose the greatest threat in textile wet processing are alkaline earth and heavy metal contaminants such as iron, manganese, calcium and magnesium.

Textile wet processes comprise of desizing, scouring, bleaching, dyeing, printing and chemical finishing. Water hardness caused by calcium and magnesium impurities in cotton, may cause several problems in each of the textile wet processes. In desizing process, effectiveness of the wetting agents used may be reduced in the presence of calcium and magnesium ions. In scouring processes, calcium and magnesium ions may precipitate the soaps used, forming a sticky insoluble substance which deposits on the cotton fabric (Losonczi et al., 2005). These deposits impair the fabric handle, cause resist-spots in dyeing, attract soil to the material and cause inconsistent absorbency in subsequent

*Authror for correspondence; E-mail: mumtaz@ntu.edu.pk processes. Although most synthetic detergents today used in scouring do not precipitate in the presence of calcium and magnesium ions, the fatty acid hydrolysis products formed by the saponification of natural waxes, fats, and oils in the fibres will precipitate. Formation of complexes with alkaline and alkaline earth salts drastically reduces the solubility and the rate of dissolution of surfactants, thus impairing the wash removal ability of the surfactants (Bille, 1987).

Although magnesium produces beneficial effects when present in hydrogen peroxide bleaching solutions, the presence of calcium may result in decreased stability of peroxide bath due to blockage of stabilisers, harsh handle of the fabric due to deposition of insoluble salts and decrease in fabric whiteness due to formation of insoluble products with optical brighteners. In bleaching cotton with hydrogen peroxide, sodium silicate is used as stabiliser, which may be converted to calcium silicate in the presence of calcium impurities. This calcium silicate has poor water solubility and is not washed-off the fabric easily, resulting in harsh fabric handle (Topalovic, 2007).

In dyeing, the presence of calcium and magnesium ions may result in lowering of solubility of dyes and staining due to formation of insoluble products with dye, change in dyeing shades, and difficulties in the removal of hydrolysed reactive dye ensuing in low washing fastness. Most water-soluble anionic dyes are sodium salts of sulphonic acid which, in the pre-sence of calcium and magnesium impurities, may be converted into their respective salts with lower water solubility, and thus staining. In the washingoff stage, the removal of unfixed hydrolysed reac- 
tive dye is also reduced in the presence of calcium impurities (Akcakoca et al., 2007).

Due to so many problems associated with the calcium and the magnesium ions, their absence is imperative during wet processing of cotton material. It is usually suspected that the major source of these impurities is the water used in textile processing. However, this may not always be the case, as these impurities may also come with the cotton itself (Hashem, 2007). The impact of these impurities has been explained above. In order to prevent the deleterious effect of calcium and magnesium impurities in cotton, an additional demineralisation process may become necessary leading to an extra burden on the processing cost. Alternatively, suitable sequestering agents may be used to alleviate the problems associated with calcium and magnesium impurities in cotton. However, this again adds to the processing cost. The present study was undertaken to compare the hardness characteristics of various global varieties of cotton to determine how much these different varieties contribute to the hardness of water used in the textile processing.

\section{Materials and Methods}

Samples of nineteen varieties of cotton fibres originating from different countries were obtained. Four of them were from the USA (SJV Pima, Elpaso, Memphis, Mote), three each from Egypt (Giza 70, Giza 86, Giza 88) and Brazil (Lot 992, Lot 1017, Lot 1832), two each from India (MCU 5, Shankar 6) and Commonwealth of Independent States (Elisa, Sultop), and one each from Mali, Greece, Ivory Coast and Pakistan. As all the collected samples were in raw form, they contained varied content of trash such as dust, seed-coat fragments, leaves, and stems etc. In order to eliminate the influence of these impurities on the test results, the non-lint trash content of each sample of cotton was removed by passing the samples through a laboratory machine "Shirley Analyzer" as per standard method (ASTM D2812-07, 2009). The machine operates on mechanicalpneumatic principles and segregates trash and cotton fibres in separate chambers.

After the preliminary removal of trash, samples of each variety of cotton were conditioned as per standard method (ASTM D1776-08, 2009) and the representative specimens of $10 \mathrm{~g}$ of each cotton variety were boiled separately in $500 \mathrm{~mL}$ of distilled water. Initial hardness of the distilled water used was zero. After 10 min of boiling in distilled water, cotton fibres were removed from water with tweezers and the excess liquid was allowed to drip back to the extract. The extract was allowed to cool down at room temperature. Then the hardness of each extract was determined according to the method described elsewhere (Vogel and Bassett, 1999).

\section{Results and Discussion}

The hardness characteristics of different varieties of cotton tested in this study are presented in tabular and graphic forms (Table 1 and Fig. 1).

As can be seen, CIS Elisa cotton was found to be the best in terms of hardness characteristics, resulting in absolutely zero water hardness. The worst among the tested varieties, in terms of total hardness, was Brazil Lot 992, while Pakistani cotton was only the second worst. However, Pakistani cotton was found to contribute the highest towards the magnesium hardness of water rather than the calcium hardness. Since presence of magnesium is beneficial in hydrogen peroxide bleaching, it means that Pakistani cotton behaves the best as far as bleaching process is concerned. However, along with calcium, magnesium may contribute to other

Table 1. Hardness of different varieties of cotton

\begin{tabular}{lllll}
\hline Cotton varieties & Country of & \multicolumn{2}{l}{ Calcium } & \multicolumn{2}{l}{ Magnesium Total } \\
origin & $\begin{array}{l}\text { hardness } \\
\text { hardness } \\
\text { (ppm) }\end{array}$ & $\begin{array}{l}\text { hardness } \\
(\mathrm{ppm})\end{array}$ \\
\hline Elisa & Uzbekistan & 0 & 0 & 0 \\
Shankar 6 & India & 18 & 17 & 35 \\
Mote & USA & 23 & 14 & 37 \\
MCU-5 & India & 21 & 17 & 38 \\
Mali & Mali & 33 & 7 & 40 \\
Barkat & Sudan & 26 & 15 & 41 \\
Giza-88 & Egypt & 12 & 30 & 42 \\
SJV Pima & USA & 20 & 22 & 42 \\
Brazil Lot 1832 & Brazil & 32 & 10 & 42 \\
Ivory Coast & Ivory Coast & 35 & 8 & 43 \\
Giza-86 & Egypt & 18 & 28 & 46 \\
Sultop & Uzbekistan & 18 & 29 & 47 \\
Giza-70 & Egypt & 31 & 21 & 52 \\
Elpaso & USA & 29 & 26 & 55 \\
Memphis & USA & 34 & 22 & 56 \\
Brazil Lot 1017 & Brazil & 45 & 13 & 58 \\
Greece & Greece & 52 & 14 & 66 \\
CIM-443 & Pak & 27 & 47 & 74 \\
Brazil Lot 992 & Brazil & 77 & 2 & 79 \\
\hline \hline
\end{tabular}




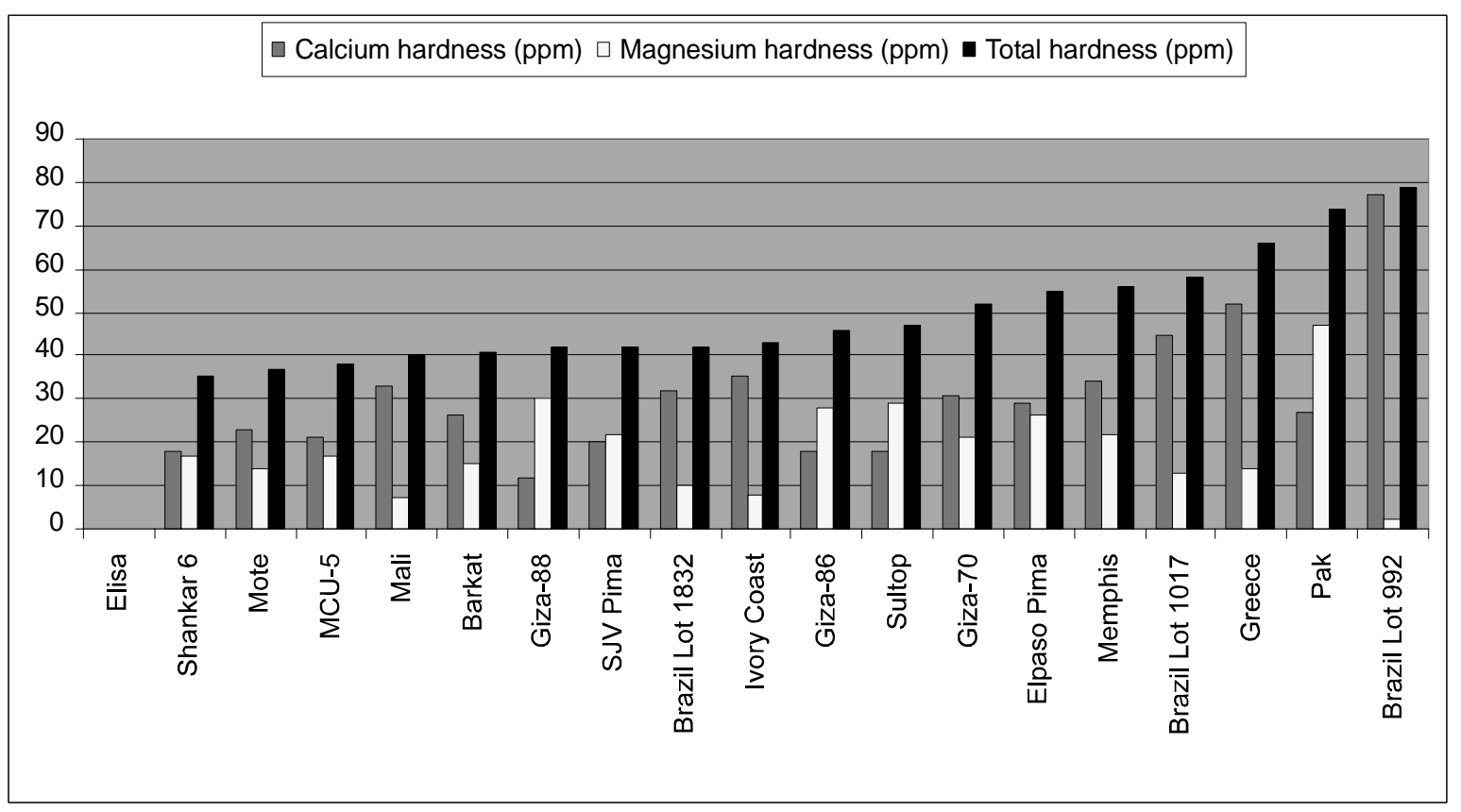

Fig. 1. Hardness characteristics of different varieties of cotton.

problems in wet processing such as decreased efficiency of desizing and scouring as well as irregularities in dyeing.

The results indicate that most of the cotton varieties have significant amount of salts of calcium and magnesium, which can contribute to hardness of the water used in wet processing. The ratio of the water and cotton used in this study was 50:1. In most of the textile wet processes, much lower liquor ratio is employed. Hence, it is expected that at lower level of liquor ratio, the contribution of cotton to water hardness may be much higher. This means that water softening plants alone are not sufficient to guarantee that no problem associated with water hardness will occur during textile wet processing since the salts causing water hardness may also come with the cotton even when initially the water is absolutely free from the calcium and magnesium ions. Therefore, to be on the safe side, it is always advisable to subject cotton either to a demineralization process in order to remove the naturally occurring hardness-causing salts of calcium and magnesium or to make use of sequestering agents during textile wet processing (Strohlen, 2007).

\section{Conclusion}

The level of impurities such as calcium and magnesium is different in different global varieties of cotton. Due to the presence of calcium and magnesium salts, different varieties of cotton contribute to water hardness in different degrees. Calcium and magnesium impurities present in cotton may cause several problems in textile wet processing, including reduction in the efficiency of desizing, scouring and bleaching operations, shade change and staining in dyeing and poor washing fastness of dyed and printed fabrics. In order to avoid these problems in textile wet processing, appropriate measures must be taken such as demineralization of cotton or use of sequestering agents during wet processing even when soft water is used for processing.

\section{References}

Akcakoca, E.P., Ozguney, A.T., Atav, R. 2007. The efficiency of washing agents in the post-dyeing removal of hydrolyzed reactive dye. Dyes and Pigments, 72: 23-27.

ASTM D1776-08, 2009. Standard Practice for Conditioning and Testing Textiles, Annual Book of ASTM Standards, vol. 07.01, 2009.

ASTM D2812-07, 2009. Standard Test Method for Non-Lint Content of Cotton, Annual Book of ASTM Standards, vol. 07.01, 2009.

Becerra, C.A. 2000. World Cotton Demand in the Future: Issues on Competitiveness, $25^{\text {th }}$ International Cotton Conference, Bremen, Germany. 
Bille, H.E. 1987. Correct pretreatment - the first step to quality in modern textile processing. Journal of Society of Dyers and Colourists, 12: 427-434.

Cook, J.G. 1984. Handbook of Textile Fibres, Natural Fibres, vol.1, $5^{\text {th }}$ edition, Merrow, UK.

Hashem, M.M. 2007. An approach towards a single pretreatment recipe for different types of cotton. Fibers and Textiles in Eastern Europe, 15: 85-92.

Lewin, M. 2006. Handbook of Fiber Chemistry, $3^{\text {rd }}$ edition, CRC Press, Boca Raton, Florida, USA.

Losonczi, A., Csiszar, E., Szakacs, G., Bezur, L. 2005. Role of the EDTA chelating agent in bioscouring of cotton. Textile Research Journal, 75: 411-417.

Strohlen, J. 2007. Demineralisation of woven fabric with hydrochloric acid. Asian Dyer, 4: 50-51.

Topalovic, T. Nierstrasz, V.A., Bautista, L., Jocic, D., Navarro, A., Warmoeskerken, M.M.C.G. 2007. Analysis of the effects of catalytic bleaching on cotton. Cellulose, 14:385-400.

Vogel, A.I., Bassett, J. 1978. Vogel's Textbook of Quantitative Inorganic Analysis, Including Elementary Instrumental Analysis, A.I. Vogel and J. Basset (eds.), 925 pp., $4^{\text {th }}$ edition, Longman, New York, USA.

Wilson, C. 2006. Cotton, 320 pp., $1^{\text {st }}$ edition, Mariner Books, USA.

Yafa, S. 2006. Cotton: The Biography of a Revolutionary Fiber, Penguin, Books, New York, USA. 